\title{
Asymptotic Expansion of Wavelet Transform
}

\author{
Ashish Pathak', Prabhat Yadav ${ }^{2}$, Madan Mohan Dixit ${ }^{2}$ \\ ${ }^{1}$ Department of Mathematics \& Statistics, Dr. Harisingh Gour Central University, Sagar, India \\ ${ }^{2}$ Department of Mathematics, North Eastern Regional Institute of Science and Technology (NERIST), Nirjuli, India \\ Email: pathak maths@yahoo.com,yprabhat9@gmail.com,mmdixit1975@yahoo.co.in
}

Received 14 October 2014; revised 30 October 2014; accepted 14 November 2014

Copyright (C) 2015 by authors and Scientific Research Publishing Inc.

This work is licensed under the Creative Commons Attribution International License (CC BY).

http://creativecommons.org/licenses/by/4.0/

\section{(c) (i) Open Access}

\section{Abstract}

In the present paper, we obtain asymptotic expansion of the wavelet transform for large value of dilation parameter a by using López technique. Asymptotic expansion of Shannon wavelet, Morlet wavelet and Mexican Hat wavelet transform are obtained as special cases.

\section{Keywords}

\section{Asymptotic Expansion, Wavelet Transform, Mellin Convolution, Integral Transform}

\section{Introduction}

The continuous wavelet transform of a function $h$ with respect to the wavelet $\phi$ is defined as

$$
\left(W_{\phi} h\right)(b, a)=a^{\frac{-1}{2}} \int_{\mathbb{R}} h(x) \overline{\phi\left(\frac{x-b}{a}\right)} \mathrm{d} x, \quad a>0, \quad b \in \mathbb{R},
$$

provided the integral exists [1]. The asymptotic expansion for Mellin convolution

$$
I(\mu)=\int_{0}^{\infty} h(x) g(\mu x) \mathrm{d} x ; \quad \text { as } \quad \mu \rightarrow 0+,
$$

was proposed by López [2], under dyadic conditions on $g$ and $h$. Let us remind earlier results from [2], which will be used in present study. We assume that $g(x)$ and $h(x)$ have asymptotic expansions of the form:

$$
g(x)=\sum_{i=0}^{n-1} a_{i} x^{i-p}+g_{n}(x) ; \quad \text { as } \quad x \rightarrow 0+,
$$

and

$$
h(x)=\sum_{i=0}^{n-1} b_{i} x^{-i-q}+h_{n}(x) ; \quad \text { as } \quad x \rightarrow \infty .
$$


Also assume that

$$
g(x)=O\left(x^{-\lambda_{1}}\right) \text {; when } x \rightarrow \infty \text { and } \lambda_{1} \in \mathbb{R} \text {, }
$$

and

$$
h(x)=O\left(x^{-\lambda_{2}}\right) \text {; when } x \rightarrow 0+\text { and } \lambda_{2} \in \mathbb{R},
$$

with the parameters $p, q, \lambda_{1}$ and $\lambda_{2}$ satisfying the following conditions:

$$
p+\lambda_{1}<1<q+\lambda_{2} ; \quad \lambda_{1}<q \text { and } p<\lambda_{2} .
$$

The asymptotic expansion of (2) at the origin is given by the following Theorem ([2], pp. 631, 633, 634).

Theorem 1 Assume that $(i) g(x)$ and $h(x)$ are locally integrable on $(0, \infty)$; (ii) $g(x)$ and $h(x)$ have expansions of the form (3), (5) and (4), (6) respectively and (iii) p, q, $\lambda_{1}$ and $\lambda_{2}$ satisfy (7), then the asym- ptotic expansion of (2) as $\mu \rightarrow 0+$ are given by

Case I: For any $n=1,2,3, \cdots$ and $m=n+\lfloor p+q\rfloor$ with $p+q \notin \mathbb{Z}$, we have

$$
\int_{0}^{\infty} h(x) g(\mu x) \mathrm{d} x=\sum_{i=0}^{n-1} b_{i} M[g ; 1-i-q] \mu^{i+q-1}+\sum_{i=0}^{m-1} a_{i} M[h ; 1+i-p] \mu^{i-p}+O\left(\mu^{n+q-1}\right) .
$$

Case II: For any $n=1,2,3, \cdots$ and $m=n+p+q-1$ with $p+q \in \mathbb{N}$, we have

$$
\begin{aligned}
\int_{0}^{\infty} h(x) g(\mu x) \mathrm{d} x= & \sum_{i=0}^{p+q-2} a_{i} M[h ; 1+i-p] \mu^{i-p} \\
& +\sum_{i=0}^{n-1} \mu^{i+q-1}\left\{-b_{i} a_{i+p+q-1} \log \mu+\lim _{z \rightarrow 0}\left[b_{i} M[g ; z+1-i-q]+a_{i+p+q-1} M[h ; z+i+q]\right]\right\} \\
& +O\left(\mu^{m-p} \log \mu\right) .
\end{aligned}
$$

Case III: For any $n=1,2,3, \cdots$ and $n=m+1-p-q$ with $1-p-q \in \mathbb{N}$, we have

$$
\begin{aligned}
\int_{0}^{\infty} h(x) g(\mu x) \mathrm{d} x= & \sum_{i=0}^{-p-q} b_{i} M[g ; 1-i-q] \mu^{i+q-1} \\
& +\sum_{i=0}^{m-1} \mu^{i-p}\left\{-a_{i} b_{i+1-p-q} \log \mu+\lim _{z \rightarrow 0}\left[b_{i+1-p-q} M[g ; z+p-i]+a_{i} M[h ; z+i+1-p]\right]\right\} \\
& +O\left(\mu^{m-p} \log \mu\right) .
\end{aligned}
$$

By using Wong technique, the asymptotic expansions of wavelet transform (1) for large and small values of dilation parameters and translation, parameters were obtained by Pathak and Pathak 2009 [3]-[5].

The main aim of the present paper is to derive asymptotic expansion of the wavelet transform for large value of a, by using Theorem 1. We also obtain asymptotic expansions for the special transforms corresponding to Shannon wavelet, Morlet wavelet and Mexican hat wavelet.

\section{Asymptotic Expansion of the Wavelet Transform for Large Value of $a$}

In this section, we obtain asymptotic expansion of the wavelet transform (1), when $a \rightarrow \infty$.

Now, let us rewrite (1) in the form:

$$
\begin{aligned}
\left(W_{\phi} h\right)(b, a) & =\vartheta^{\frac{1}{2}} \int_{-\infty}^{\infty} h(x+b) \overline{\phi(\vartheta x)} \mathrm{d} x \\
& =\vartheta^{\frac{1}{2}}\left\{\int_{0}^{\infty} h(x+b) \overline{\phi(\vartheta x)} \mathrm{d} x+\int_{0}^{\infty} h(-x+b) \overline{\phi(-\vartheta x)} \mathrm{d} x\right\} \\
& =\left(W_{\phi}^{+} h\right)(b, a)+\left(W_{\phi}^{-} h\right)(b, a)(\text { say }) ;
\end{aligned}
$$

where, $\vartheta=\frac{1}{a}$ and $\mathrm{b}$ is assumed to be a fixed real number. 
Setting $h(x+b)=\chi(x)$ and assume that $\chi(x)$ and $\overline{\phi(x)}$ are locally integrable on $(0, \infty)$. Further assume that $\chi(x)$ and $\overline{\phi(x)}$ have asymptotic expansions of the form

$$
\begin{gathered}
\overline{\phi(x)}=\sum_{i=0}^{n-1} c_{i} x^{i-p}+\overline{\phi_{b}(x)} ; \quad \text { as } \quad x \rightarrow 0+, \\
\chi(x)=\sum_{i=0}^{n-1} d_{i} x^{-i-q}+\chi_{n}(x) ; \quad \text { as } \quad x \rightarrow \infty .
\end{gathered}
$$

Also assume that

$$
\overline{\phi(x)}=O\left(x^{-\beta}\right) ; \quad \text { as } x \rightarrow \infty, \beta \in \mathbb{R},
$$

and

$$
\chi(x)=O\left(x^{-\gamma}\right) ; \quad \text { as } x \rightarrow 0+, \quad \gamma \in \mathbb{R} .
$$

with the parameters $p, q, \beta$ and $\gamma$ satisfy the following condition

$$
p+\beta<1<q+\gamma ; \quad \beta<q \text { and } p<\gamma .
$$

Then by using, Theorem 1 , we obtain asymptotic expansion of $\left(W_{\phi}^{+} h\right)(b, a)$ for large value of a.

Case I: When $n=1,2,3, \cdots$ and $m=n+\lfloor p+q\rfloor$ with $p+q \notin \mathbb{Z}$, we have

$$
\begin{aligned}
\left(W_{\phi}^{+} h\right)(b, a) & =\sum_{i=0}^{n-1} b_{i} M[\bar{\phi} ; 1-i-q] \vartheta^{i+q-1 / 2}+\sum_{i=0}^{m-1} a_{i} M[\chi ; 1+i-p] \vartheta^{i-p+1 / 2}+O\left(\vartheta^{n+q-1 / 2}\right) \\
& =\sum_{i=0}^{n-1} b_{i} M[\bar{\phi} ; 1-i-q] a^{-i-q+1 / 2}+\sum_{i=0}^{m-1} a_{i} M[\chi ; 1+i-p] a^{-i+p-1 / 2}+O\left(a^{-n-q+1 / 2}\right) .
\end{aligned}
$$

Case II: When $n=1,2,3, \cdots$ and $m=n+p+q-1$ with $p+q \in \mathbb{N}$, we have

$$
\begin{aligned}
& \left(W_{\phi}^{+} h\right)(b, a)=\sum_{i=0}^{p+q-2} a_{i} M[\chi ; 1+i-p] \vartheta^{i-p+1 / 2} \\
& +\sum_{i=0}^{n-1} \vartheta^{i+q-1 / 2}\left\{-b_{i} a_{i+p+q-1} \log \vartheta+\lim _{z \rightarrow 0}\left[b_{i} M[\bar{\phi} ; z+1-i-q]+a_{i+p+q-1} M[\chi ; z+i+q]\right]\right\}+O\left(\vartheta^{m-p+1 / 2} \log \vartheta\right) \\
& =\sum_{i=0}^{p+q-2} a_{i} M[\chi ; 1+i-p] a^{-i+p-1 / 2} \\
& +\sum_{i=0}^{n-1} a^{-i-q+1 / 2}\left\{-b_{i} a_{i+p+q-1} \log (1 / a)+\lim _{z \rightarrow 0}\left[b_{i} M[\bar{\phi} ; z+1-i-q]+a_{i+p+q-1} M[\chi ; z+i+q]\right]\right\}+O\left(a^{-m+p-1 / 2} \log (1 / a)\right) .
\end{aligned}
$$

Case III: When $m=1,2,3, \cdots$ and $n=m+1-p-q$ with $1-p-q \in \mathbb{N}$, we have

$$
\begin{aligned}
\left(W_{\phi}^{+} h\right)(b, a)= & \sum_{i=0}^{-p-q} b_{i} M[\bar{\phi} ; 1-i-q] \vartheta^{i+q-1 / 2} \\
& +\sum_{i=0}^{m-1} \vartheta^{i-p+1 / 2}\left\{-a_{i} b_{i+1-p-q} \log \vartheta+\lim _{z \rightarrow 0}\left[b_{i+1-p-q} M[\bar{\phi} ; z+p-i]+a_{i} M[\chi ; z+i+1-p]\right]\right\} \\
& +O\left(\vartheta^{m-p+1 / 2} \log \vartheta\right) \\
= & \sum_{i=0}^{-p-q} b_{i} M[\bar{\phi} ; 1-i-q] a^{-i-q+1 / 2} \\
& +\sum_{i=0}^{m-1} a^{-i+p-1 / 2}\left\{-a_{i} b_{i+1-p-q} \log (1 / a)+\lim _{z \rightarrow 0}\left[b_{i+1-p-q} M[\bar{\phi} ; z+p-i]+a_{i} M[\chi ; z+i+1-p]\right]\right\} \\
& +O\left(a^{-m+p-1 / 2} \log (1 / a)\right) .
\end{aligned}
$$

Similarly, we can also obtain the asymptotic expansion of $\left(W_{\phi}^{-} h\right)(b, a)$ as $a \rightarrow-\infty$. 


\section{Application}

In this section, we apply the previous result and obtain the asymptotic expansions of Shannon wavelet transform, Morlet Wavelet transform and Mexican hat wavelet transform.

\subsection{Asymptotic Expansion of the Shannon Wavelet Transform}

Let us consider $\phi$ to be Shannon wavelet and it is given by [1] $\phi(x)=\frac{\sin \left(\frac{\pi x}{2}\right)}{\left(\frac{\pi x}{2}\right)} \cos \left(\frac{3 \pi x}{2}\right)$. Since, $\phi$ is locally integrable on $(0, \infty)$ and has the asymptotic expansion:

$$
\overline{\phi(x)}=1-\frac{7 \pi^{2} x^{2}}{6}+\frac{31 \pi^{4} x^{4}}{120}-\frac{217 \pi^{6} x^{6}}{5040}+O\left(x^{7}\right) \quad \text { as } x \rightarrow 0+
$$

with

$$
\overline{\phi(x)}=O(1) ; \quad \text { as } \quad x \rightarrow \infty .
$$

Consider, $\chi(x)$ is locally integrable on $(0, \infty)$ and satisfies (13) and (15) with parameters

$$
1<q+\gamma ; \quad q>0 \text { and } 0<\gamma .
$$

Now, by using (17), (18) and (19) respectively and by means of formula ([6], p. 321, (41)), then the asymptotic expansions of Shannon wavelet transform are given by

Case I: When $m=7+\lfloor q\rfloor$ and $q \notin \mathbb{Z}$, we get

$$
\begin{aligned}
\left(W_{\phi}^{+} h\right)(b, a)=\sum_{i=0}^{6} b_{i} & \left\{\left(1-2^{i+q}\right) \pi^{-1+i+q} \cos \left[\frac{1}{2} \pi(-1+i+q)\right] \Gamma[-i-q]\right\} a^{-i-q+1 / 2} \\
& +\sum_{i=0}^{m-1} a_{i} M[\chi ; 1+i] a^{-i-1 / 2}+O\left(a^{-13 / 2-q}\right) .
\end{aligned}
$$

Case II: When $m=6+q$ and $q \in \mathbb{N}$, we get

$$
\begin{aligned}
\left(W_{\phi}^{+} h\right)(b, a)= & \sum_{i=0}^{q-2} a_{i} M[\chi ; 1+i] a^{-i-1 / 2}+\sum_{i=0}^{6} a^{-i-q+1 / 2}\left\{-b_{i} a_{i+q-1} \log (1 / a)\right. \\
& \left.+\lim _{z \rightarrow 0}\left[b_{i}\left\{\left(1-2^{i+q-z}\right) \pi^{-1+i+q-z} \cos \left[\frac{1}{2} \pi(-1+i+q-z)\right] \Gamma[-i-q+z]\right\}+a_{i+q-1} M[\chi ; z+i+q]\right]\right\} \\
& +O\left(a^{-m-1 / 2} \log (1 / a)\right) .
\end{aligned}
$$

Case III: When $m=6+q$ and $1-q \in \mathbb{N}$, we get

$$
\begin{aligned}
\left(W_{\phi}^{+} h\right)(b, a)= & \sum_{i=0}^{-q} b_{i}\left\{\left(1-2^{i+q}\right) \pi^{-1+i+q} \cos \left[\frac{1}{2} \pi(-1+i+q)\right] \Gamma[-i-q]\right\} a^{-i-q+1 / 2}+\sum_{i=0}^{m-1} a^{-i-1 / 2}\left\{-a_{i} b_{i+1-q} \log (1 / a)\right. \\
& \left.+\lim _{z \rightarrow 0}\left[b_{i+1-q}\left\{\left(1-2^{i+q-z}\right) \pi^{-1+i+q-z} \cos \left[\frac{1}{2} \pi(-1+i+q-z)\right] \Gamma[-i-q+z]\right\}+a_{i} M[\chi ; z+i+1]\right]\right\} \\
& +O\left(a^{-m-1 / 2} \log (1 / a)\right) .
\end{aligned}
$$

\subsection{Asymptotic Expansion of the Morlet Wavelet Transform}

We choose $\phi$ to be Morlet wavelet and it is given by [1] $\phi(x)=\mathrm{e}^{i \omega_{0} x-\frac{x^{2}}{2}}$. Since, $\phi$ is locally integrable on 
$(0, \infty)$ and has the asymptotic expansion as

$$
\overline{\phi(x)}=1-i w_{o} x+\left(\frac{-1}{2}-\frac{w_{o}^{2}}{2}\right) x^{2}+\frac{1}{6} i\left(3 w_{o}+w_{o}^{3}\right) x^{3}+O\left(x^{4}\right) ; \quad \text { as } \quad x \rightarrow 0+,
$$

with

$$
\overline{\phi(x)}=O(1) ; \text { as } x \rightarrow \infty \text {. }
$$

Let $\chi(x)$ is locally integrable on $(0, \infty)$ and satisfy (13) and (15) with parameters (22). Now by using (17), (18) and (19) respectively and by formula ([6], pp. 318, 320, $(10,30)$ ), then the asymptotic expansions of Morlet wavelet transform are given by

Case I: When $m=4+\lfloor q\rfloor$ and $q \notin \mathbb{Z}$, we have

$$
\begin{aligned}
\left(W_{\phi}^{+} h\right)(b, a)= & \sum_{i=0}^{3} b_{i}\left\{2 ^ { \frac { 1 } { 2 } ( - 1 - i - q ) } \left(\Gamma\left[\frac{1}{2}(1-i-q)\right]_{1} F_{1}\left[\frac{1}{2}(1-i-q), \frac{1}{2},-\frac{w_{o}^{2}}{2}\right]\right.\right. \\
& \left.+i \sqrt{2} \Gamma\left[1-\frac{i}{2}-\frac{q}{2}\right]_{1} F_{1}\left[1-\frac{i}{2}-\frac{q}{2}, \frac{3}{2},-\frac{w_{o}^{2}}{2}\right] w_{o}\right\} a^{-i-q+\frac{1}{2}} \\
& +\sum_{i=0}^{m-1} a_{i} M[\chi ; 1+i] a^{-i-\frac{1}{2}}+O\left(a^{-7 / 2-q}\right) .
\end{aligned}
$$

Case II: When $m=3+q$ and $q \in \mathbb{N}$, we have

$$
\begin{aligned}
& \left(W_{\phi}^{+} h\right)(b, a)=\sum_{i=0}^{q-2} a_{i} M[\chi ; 1+i] a^{-i-1 / 2} \\
& +\sum_{i=0}^{3} a^{-i-q+1 / 2}\left\{-b_{i} a_{i+q-1} \log (1 / a)+\lim _{z \rightarrow 0}\left[b _ { i } \left\{2 ^ { \frac { 1 } { 2 } ( - 1 - i - q + z ) } \left(\Gamma\left[\frac{1}{2}(1-i-q+z)\right]_{1} F_{1}\left[\frac{1}{2}(1-i-q+z), \frac{1}{2},-\frac{w_{o}^{2}}{2}\right]\right.\right.\right.\right. \\
& \left.\left.\left.\left.+i \sqrt{2} \Gamma\left[1-\frac{i}{2}-\frac{q}{2}+\frac{z}{2}\right]_{1} F_{1}\left[1-\frac{i}{2}-\frac{q}{2}+\frac{z}{2}, \frac{3}{2},-\frac{w_{o}^{2}}{2}\right] w_{o}\right)\right\}\right]+a_{i+q-1} M[\chi ; z+i+q]\right\} \\
& +O\left(a^{-m-1 / 2} \log (1 / a)\right) .
\end{aligned}
$$

Case III: When $m=3+q$ and $1-q \in \mathbb{N}$, we have

$$
\begin{aligned}
& \left(W_{\phi}^{+} h\right)(b, a)= \\
& \sum_{i=0}^{-q} b_{i}\left\{2^{\frac{(-1-i-q)}{2}}\left(\Gamma\left[\frac{1}{2}(1-i-q)\right]_{1} F_{1}\left[\frac{1}{2}(1-i-q), \frac{1}{2},-\frac{w_{o}^{2}}{2}\right]+i \sqrt{2} \Gamma\left[1-\frac{i}{2}-\frac{q}{2}\right]_{1} F_{1}\left[1-\frac{i}{2}-\frac{q}{2}, \frac{3}{2},-\frac{w_{o}^{2}}{2}\right] w_{o}\right)\right\} a^{-i-q+\frac{1}{2}} \\
& +\sum_{i=0}^{m-1} a^{-i-1 / 2}\left\{-a_{i} b_{i+1-q} \log (1 / a)+\lim _{z \rightarrow 0}\left[b _ { i + 1 - q } \left\{2 ^ { \frac { 1 } { 2 } ( - 1 - i - q + z ) } \left(\Gamma\left[\frac{1}{2}(1-i-q+z)\right]_{1} F_{1}\left[\frac{1}{2}(1-i-q+z), \frac{1}{2},-\frac{w_{o}^{2}}{2}\right]\right.\right.\right.\right. \\
& \left.\left.\left.\left.+i \sqrt{2} \Gamma\left[1-\frac{i}{2}-\frac{q}{2}+\frac{z}{2}\right]_{1} F_{1}\left[1-\frac{i}{2}-\frac{q}{2}+\frac{z}{2}, \frac{3}{2},-\frac{w_{o}^{2}}{2}\right] w_{o}\right)\right\}+a_{i} M[\chi ; z+i+1]\right]\right\}+O\left(a^{-m-1 / 2} \log (1 / a)\right) .
\end{aligned}
$$

\subsection{Asymptotic Expansion of the Mexican Hat Wavelet Transform}

We choose $\phi$ to be Mexican hat wavelet $\phi(x)=\left(1-x^{2}\right) \mathrm{e}^{\frac{-x^{2}}{2}}$ [1]. Since $\phi$ is locally integrable on $(0, \infty)$ 
and has the asymptotic expansion:

$$
\overline{\phi(x)}=1-\frac{3 x^{2}}{2}+\frac{5 x^{4}}{8}-\frac{7 x^{6}}{48}+\frac{3 x^{8}}{128}+O\left(x^{9}\right) ; \quad \text { as } \quad x \rightarrow 0+
$$

with

$$
\overline{\phi(x)}=O(1) ; \quad \text { as } x \rightarrow \infty
$$

As $\chi(x)$ is locally integrable on $(0, \infty)$ and satisfies (13) and (15) with parameters (22). Now by using (17), (18) and (19) respectively, we can obtain the asymptotic expansion of Mexican hat wavelet transform by using formula ([6], p. 313, (13))

Case I: When $m=9+\lfloor q\rfloor$ and $q \notin \mathbb{Z}$, we have

$$
\left(W_{\phi}^{+} h\right)(b, a)=\sum_{i=0}^{8} b_{i}\left\{2^{2^{\frac{1}{2}(-1-i-q)}}(i+q) \Gamma\left[\frac{1}{2}(1-i-q)\right]\right\} a^{-i-q+\frac{1}{2}}+\sum_{i=0}^{m-1} a_{i} M[\chi ; 1+i] a^{-i-\frac{1}{2}}+O\left(a^{-17 / 2-q}\right) .
$$

Case II: When $m=8+q$ and $q \in \mathbb{N}$, we have

$$
\begin{aligned}
& \left(W_{\phi}^{+} h\right)(b, a)=\sum_{i=0}^{q-2} a_{i} M[\chi ; 1+i] a^{-i-1 / 2} \\
& +\sum_{i=0}^{8} a^{-i-q+1 / 2}\left\{-b_{i} a_{i+q-1} \log (1 / a)+\lim _{z \rightarrow 0}\left[b_{i}\left\{2^{\frac{1}{2}(-1-i-q+z)}(i+q-z) \Gamma\left[\frac{1}{2}(1-i-q+z)\right]\right\}\right]+a_{i+q-1} M[\chi ; z+i+q]\right\}( \\
& +O\left(a^{-m-1 / 2} \log (1 / a)\right) .
\end{aligned}
$$

Case III: When $m=8+q$ and $1-q \in \mathbb{N}$, we have

$$
\begin{aligned}
& \left(W_{\phi}^{+} h\right)(b, a)=\sum_{i=0}^{-q} b_{i}\left\{2^{\frac{1}{2}(-1-i-q)}(i+q) \Gamma\left[\frac{1}{2}(1-i-q)\right]\right\} a^{-i-q+1 / 2} \\
& +\sum_{i=0}^{m-1} a^{-i-1 / 2}\left\{-a_{i} b_{i+1-q} \log (1 / a)+\lim _{z \rightarrow 0}\left[b_{i+1-q}\left\{2^{\frac{1}{2}(-1-i-q+z)}(i+q-z) \Gamma\left[\frac{1}{2}(1-i-q+z)\right]\right\}+a_{i} M[\chi ; z+i+1]\right]\right\} \\
& +O\left(a^{-m-1 / 2} \log (1 / a)\right) .
\end{aligned}
$$

\section{Acknowledgements}

The authors are thankful to Prof. R. S. Pathak, DST Center for Interdisciplinary Mathematical Sciences, Banaras Hindu University, Varanasi-221005, India, for his valuable suggestion for the improvement of the article. We thank the referee for their comments. The research of the first author was supported by U.G.C-BSR start-up grant No. F.30-12/2014 (BSR).

\section{References}

[1] Pathak, R.S. (2009) The Wavelet Transform. Atlantis Press/World Scientific, Amsterdam.

[2] López, J.L. (2007) Asymptotic Expansions of Mellin Convolutions by Means of Analytic Continuation. Journal of Computational and Applied Mathematics, 200, 628-636. http://dx.doi.org/10.1016/j.cam.2006.01.019

[3] Pathak, R.S. and Pathak, A. (2009) Asymptotic Expansion of Wavelet Transform for Small Value a. In: Pathak, R.S. and Chui, C.K., Eds., The Wavelet Transform, World Scientific, Amsterdam, 164-169.

[4] Pathak, R.S. and Pathak, A. (2009) Asymptotic Expansion of Wavelet Transform with Error Term. In: Pathak, R.S. and Chui, C.K., Eds., The Wavelet Transform, World Scientific, Amsterdam, 154-164.

[5] Pathak, R.S. and Pathak, A. (2009) Asymptotic Expansions of the Wavelet Transform for Large and Small Values of b. The International Journal of Mathematics and Mathematical Sciences, 2009, Article ID: 270492, 13 p.

[6] Erde'lyi, A., Magnus, W., Oberhettinger F. and Tricomi, F.G. (1954) Tables of Integral Transform. McGraw-Hill, New York. 